\title{
1.1.1993 Prof. Dr. med. dent. Rolf Hinz
}

DGKfo - KZV - Zahnärztekammer - BDK - Universiät Witten-Herdecke, Praxis - Fachverlag und noch mehr: Er ist dabei und jeder kennt ihn. Keineswegs ein passiver Teilnehmer, nein putzmunter, aktiv-kooperativ und mit eigenständigen Vorstellungen, Vorschlägen und Ideen. Die Teilnehmer an den Jahrestagungen der DGKfo hatten häufig Gelegenheit, ihn als Referenten zu hören. Sein besonderes Anliegen gilt der Einbeziehung technischer und zahntechnischer Entwicklungen in kieferorthopädisch-klinische Aufgaben.

Er war bereits gelernter Zahntechniker, als er sein Studium an der Ostberliner Humboldt-Universität begann, und wurde 1957 jüngster Kieferorthopäde in der damaligen DDR, bald in eigener Praxis. Ein Jahr vor dem Mauerbau wechselte Rolf Hinz in die Bundesrepublik und fing in Herne mit der Praxis von vorne an. Dazu Ämter, Aufgaben, Vorträge und viel Schriftliches: Alles nebenbei.
In einem Alter, in dem andere an den Ruhestand zu denken beginnen, hat Rolf Hinz sich habilitiert (den Lehrauftrag hatte er bereits seit zwei Jahren), und 1988 wurde er in Witten-Herdecke zum Universitätsprofessor ernannt.

Seine Frische und Vitalität sind bewundernswert. bewundernswert ist besonders, daß die Vielseitigkeit und Vielgeschäftigkeit nie zu dem Eindruck von Hetze führt: Für ihm wichtige Anliegen und Aufgaben scheint Rolf Hinz stets Zeit zu haben.

Vorstand der Deutschen Gesellschaft für Kieferorthopädie und Schriftleitung der FORTSCHRITTE DER KIEFERORTHOPÄDIE wünschen dem jungen $65 \mathrm{er}$, daß ihm Gesundheit und Frische noch viele Jahre erhalten bleiben. Und ehrlich: Im Ruhestand kann man sich Rolf Hinz auch schlecht vorstellen.

Peter Bertzbach 